\title{
COMPORTAMIENTO DEL SECADO SOLAR DE LATERITAS A LA INTEMPERIE EN CONDICIONES DE CLIMA HÚMEDO TROPICAL
}

\author{
O. B. FUENTES ${ }^{1}$, S. ESTENOZ-MEJÍA ${ }^{2}$ \\ Instituto Superior Minero Metalurgico de Moa \\ orlandobelette@gmail.com ${ }^{1}$
}

Submetido 26/02/2018 - Aceito 24/04/2020

DOI: $10.15628 /$ holos.2020.7011

\section{RESUMEN}

Las plantas niqueleras de tecnología Caron emplean el secado solar para disminuir la humedad de la mena laterítica, reflejado en el procedimiento y equipo para secado solar de materiales a la intemperie metodología de Estenoz y Espinosa (2003). El objetivo de la presente investigación consiste en evaluar el comportamiento del secado solar de lateritas a la intemperie en condiciones de clima húmedo tropical, para disminuir la humedad de la mena laterítica que procesa esta industria. El proceso de secado solar se ensayó, a escala semi-industrial, en pilas piramidales expuestas a la intemperie en condiciones de abundantes precipitaciones. Luego de 7 semanas de remoción, la humedad inicial de $37,75 \%$ se redujo hasta $26,81 \%$. El trabajo realizado sobre el secado a la intemperie redujo a valores inferiores de \$0,09 los costos por tonelada de mineral trasegado en los depósitos mineros de Punta Gorda, Moa, Holguín, Cuba.

Se obtuvo un valor promedio de humedad final equivalente a la evaporación del $40 \%$ del agua inicial. Se determinó que para alturas de pilas de 5,5 y $8,5 \mathrm{~m}$ se requieren 19 y 17 hectáreas respectivamente, para secar 4,2 millones de masa minera en base húmeda, equivalente a la producción de 30000 toneladas anuales de níquel.

PALABRAS-CLAVE: secado solar, yacimientos lateríticos, calidad de mena, pilas piramidales.

\section{SOLAR DRYING OF LATERITE IN OPEN AIR IN HUMID TROPICAL CLIMATE}

\section{ABSTRACT}

The Caron technology nickel plants use solar drying to decrease the humidity of the lateritic ore, reflected in the procedure and equipment for solar drying of materials in the open air, the methodology of Estenoz and Espinosa (2003). The objective of the present investigation consists of evaluating the behavior of the solar drying of laterites in the open air in conditions of tropical humid climate, to decrease the humidity of the lateritic ore that this industry processes. The solar drying process was tested, on a semi-industrial scale, in pyramid stacks exposed to the elements in conditions of abundant precipitation. After 7 weeks of removal, the initial humidity of $37.75 \%$ was reduced to $26.81 \%$.
\end{abstract}

The work carried out on the drying in the open air reduced to costs of less than $\$ 0.09$ the costs per ton of ore transferred in the mining deposits of Punta Gorda, Moa, Holguín, Cuba.

An average final humidity value equivalent to the evaporation of $40 \%$ of the initial water was obtained. It was determined that for heights of piles of 5.5 and 8.5 $\mathrm{m}, 19$ and 17 hectares, respectively, are required to dry 4.2 million wet mass mining, equivalent to the production of 30,000 tons of nickel per year.

KEYWORDS: solar drying, humidity, storage, mineral quality 


\section{INTRODUCCIÓN}

En los proyectos para la modernización y expansión minera de la Empresa Comandante Ernesto Che Guevara de Moa, provincia Holguín, Cuba, la definición de la tecnología en los procesos de beneficio y homogenización del mineral requieren precisar los indicadores de rendimientos y el potencial del secado solar y eólico en la mina. Sin tales parámetros, son imprecisos los diseños de las instalaciones y las tecnologías para el beneficio, la mezcla y la homogeneización de los minerales.

El desarrollo de pruebas de secado solar que considere el potencial de homogenización implícito en la tecnología de secado permitirá determinar el potencial de premezclas de las áreas destinadas al secado solar del mineral, así como determinar la capacidad del secado solar y los costos específicos de ambas operaciones durante las manipulaciones del mineral, según los procedimientos desarrollados especialmente para las condiciones mineras en la mina de la Empresa Comandante Ernesto Che Guevara (Alepuz, 2001).

El secado solar del mineral laterítico ha sido tratado por los investigadores (Alepuz, 2001), (Estenoz \& Espinosa 2003), (Estenoz, Pérez \& Rondón, 2004), (Estenoz, 2007) (Retirado, 2007, 2012 y 2015) y (Romero, 2006), donde aportaron al estudio del secado solar, y en especial (Retirado, 2012) evaluó de forma experimental la humedad durante el secado solar del mineral laterítico a la intemperie. Sin embargo, (Ricardo, \& Vargas, 2014) estudiaron la velocidad de secado del carbonato de níquel y (García, 2014) realizó una investigación sobre la evaluación de los efectos del proceso de secado sobre la calidad de los minerales lateríticos.

El objetivo de la presente investigación consiste en evaluar el comportamiento del secado solar de lateritas a la intemperie en condiciones de clima húmedo tropical, para disminuir la humedad de la mena laterítica que procesa la industria niquelera de tecnología Caron.

\section{METOdOLOGÍA}

\subsection{Acarreo}

Los experimentos se realizaron en las áreas de depósito (plazoletas) de la planta piloto del Centro de Investigación del Níquel. Se muestreó y sometió a análisis químico una masa mineral de $1700 \mathrm{t}$ guardando la proporcionalidad con el flujo de mena que ingresa al proceso fabril. Para transportar la mena, desde los frentes de minería hasta las plazoletas, se emplearon camiones Volvo A35 de $18 \mathrm{t}$ de capacidad, los cuales se pesaron vacíos y cargados para poder determinar con mayor exactitud la masa mineral (Estenoz, 2003, 2007, 2011). Se conformaron 4 pilas de mineral dirigiendo la descarga de los bultos en forma de hileras y considerando la trayectoria aparente que describe el Sol por la bóveda celeste. 


\subsection{Muestreo inicial}

Se tomaron muestras compósito en tres puntos separados entre sí por un ángulo aproximado de $90^{\circ}$, como se muestra en la Figura 1. Este procedimiento de muestreo fue validado por (García, 2014). En cada bulto de muestras tomadas en las pilas, se les determinó la composición química, el contenido de la fracción magnética, la distribución granulométrica y la humedad inicial.

\subsection{Remonte}

Las pilas se conformaron con un cargador frontal de $1,8 \mathrm{~m}^{3}$ de capacidad de la cuchara a partir de los bultos muestreados y dispuestos en hileras. Cada pila se compone de dos hileras. El remonte se realizó levantando el material hacia el centro de los bultos hasta obtener taludes planos a lo largo de la primera hilera. Lo bultos de la segunda hilera se remontaron hacia la cima de la primera, deslizándose parte del mineral sobre el talud opuesto (Marcilli, 2013).

\subsection{Remoción}

Para determinar la humedad en las pilas se realizaron muestreos diarios (a las 6:00 y a las 17:00 horas), tomando $2 \mathrm{~kg}$ de mineral en ambos taludes longitudinales de todas las pilas en remoción (durante las siete semanas de exposición al sol. Para alcanzar mayor representatividad del muestreo, se escogieron ocho zonas a lo largo de los taludes longitudinales, de manera que cada cuatro días se muestreaba en la misma zona.

En la Figura 1 los círculos discontinuos representan los contornos medios de las zonas de muestreo, distantes a 10, 20, 30 y 40 m del extremo Este de las pilas. Ocasionalmente, cuando las precipitaciones fueron muy abundantes, se realizó un muestreo en profundidad con una barrena para determinar el incremento de humedad desde la superficie del talud hasta el piso de las pilas.

Las pilas de mineral sumaron una longitud de unos $140 \mathrm{~m}$ y las mismas tuvieron una altura máxima en sus inicios de 2.7-2.9 m, con ángulo en los taludes del orden de los $60^{\circ}$. 


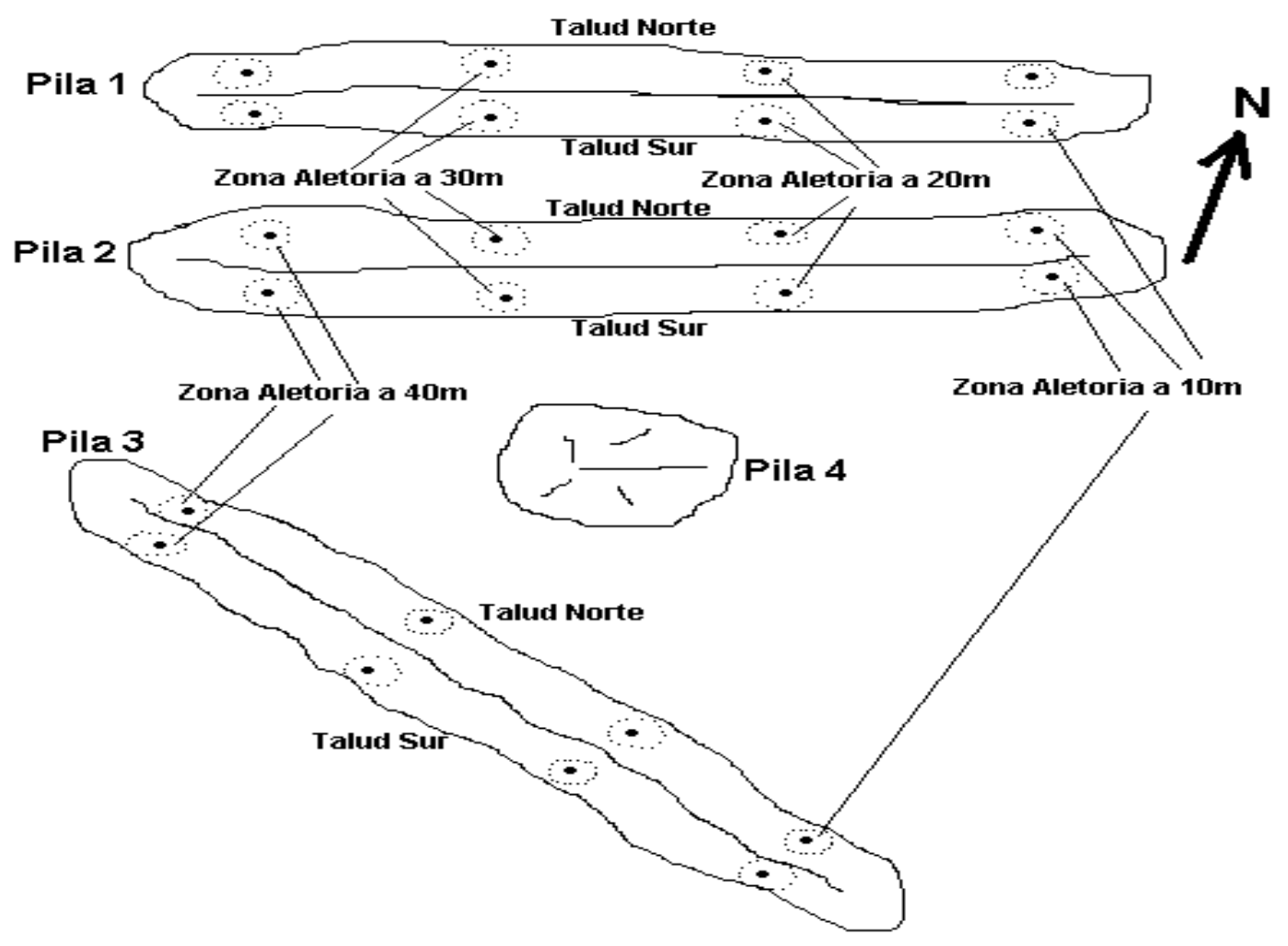

Figura 1: Distribución de las zonas de muestreo en los taludes longitudinales.

En caso de no ocurrencia de lluvias, después de cada muestreo de humedad se removió una capa de 10 a $20 \mathrm{~cm}$ de espesor, a todo lo ancho del talud Sur hacia el Norte (Figura 2). Cuando se produjeron precipitaciones, en función de la cantidad de éstas, se determinó el espesor de la capa a remover y se decidió la dirección de la remoción (sur-norte o norte-sur), según el procedimiento de secado solar a la intemperie de Estenoz (2003, 2007).

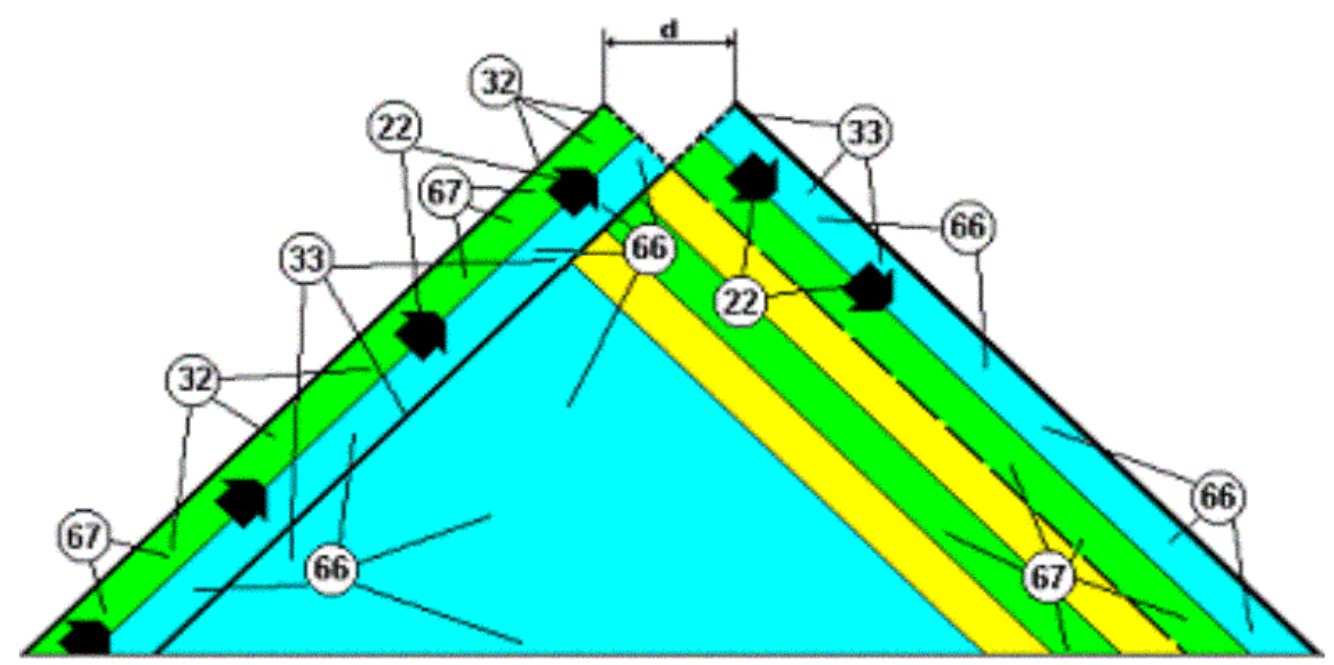

Figura 2: Esquema representativo en sección transversal de la pila durante el proceso de remoción. Leyenda: 22 dirección del desplazamiento y descenso del mineral por los taludes; 66 y 33-mineral húmedo interior o más húmedo; 67 y 32- mineral seco o menos húmedo de las capas en exposición exterior al Sol y el aire.

Para evaluar el efecto de las precipitaciones sobre las pilas, se tomaron muestras de un kilogramo como mínimo del material fino arrastrado por las aguas durante su drenaje y 
escurrimiento, y del material de las corazas formadas sobre los taludes. Las corazas comprenden el material seco que a la ocurrencia de precipitaciones se compacta o endurece en la superficie del talud e impide el paso del agua (Figura 3).

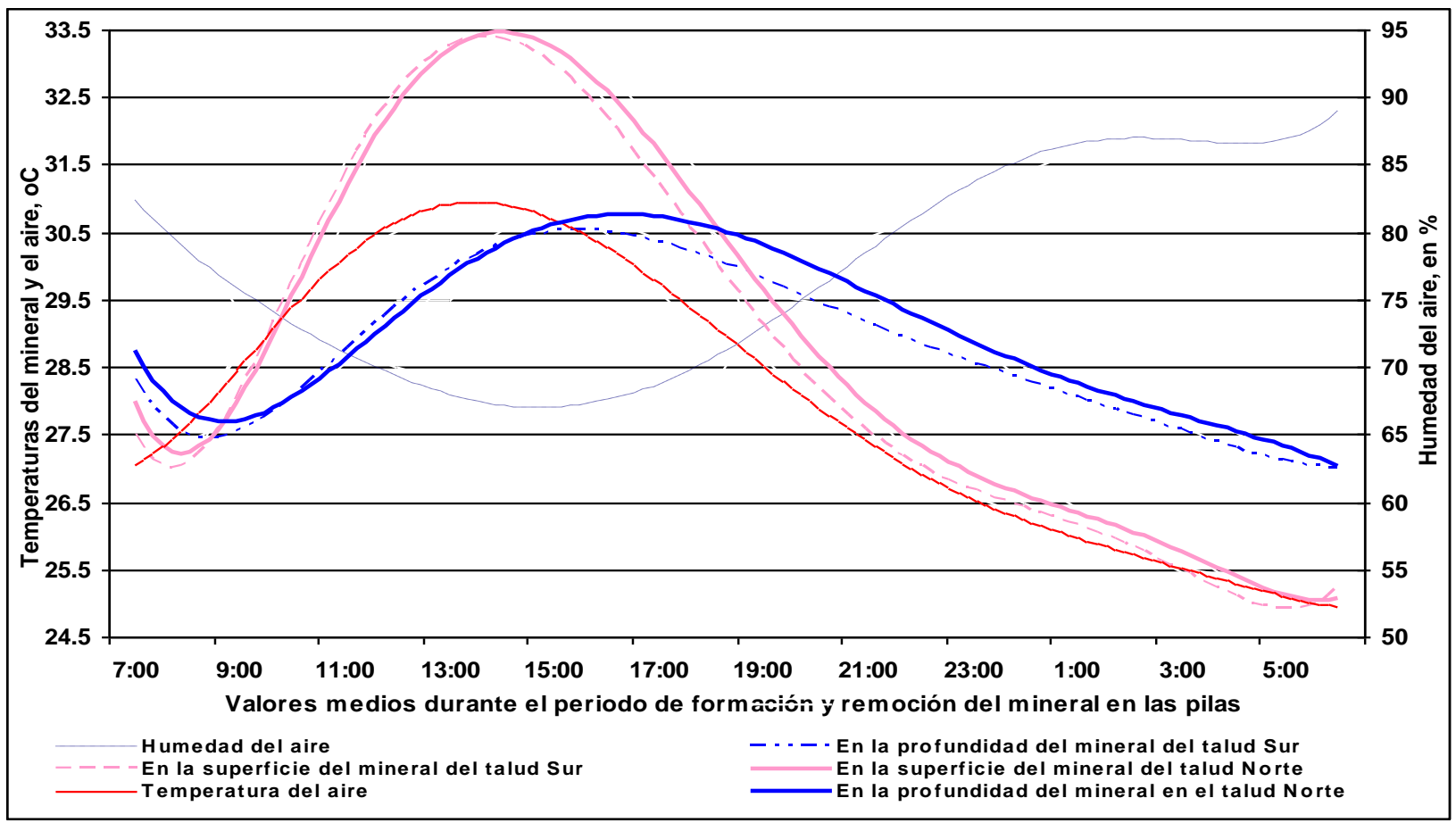

Figura 3: Comportamiento de la temperatura del mineral en función de la humedad y temperatura del aire durante el proceso de remoción.

\subsection{Monitoreo meteorológico y procesamiento de datos}

Para conocer el comportamiento de las condiciones meteorológicas del clima durante el período de la prueba, se midieron in situ las variables: radiación solar, nubosidad, humedad relativa, punto de rocío, temperatura máxima, mínima y media del aire, dirección y velocidad media y máxima predominante del viento y presión atmosférica. Las lecturas de las variables meteorológicas utilizadas para cuantificar la cantidad y los niveles de las oscilaciones de la energía solar, eólica y el calor natural del medio ambiente in situ se tomaron cada 30 min o cada una hora.

Tanto la información suministrada por la estación meteorológica del aeropuerto de Moa y por satélites geoestacionarios, uno situado sobre el Caribe Central y otro sobre las islas Bermudas (NOAA-16 y GOES-12), como las mediciones del monitoreo diario in situ, fueron procesadas mediante los programas Excel, Access y Surfer, de Microsoft.

\section{RESULTADOS Y DISCUSIÓN}

3.1 Humedad del mineral durante el proceso de remoción 
Para determinar la humedad del mineral durante el proceso de remoción, primero se realizo una caracterización del tipo de mineral presente por tipo tecnológico de mena (laterita de balance, laterita fuera de balance con contenido mayor de $1.00 \%$ ) y una humedad natural superior al $36 \%$. Los resultados obtenidos consistieron en la determinación de la reducción de la humedad del mineral desde el inicio de la prueba, durante todo el proceso de remoción y almacenamiento y hasta la evacuación total del mineral. Se dividieron los intervalos en todos los días en que ocurrieron precipitaciones, en la Tabla 1 se muestra la reducción específica de humedad en el tiempo y se consideran en cada ciclo la formación y ruptura o no de las corazas de acuerdo a los niveles de precipitaciones.

Tabla 1: Comportamiento de la humedad durante el secado solar.

\begin{tabular}{|c|c|c|c|c|c|c|c|}
\hline \multicolumn{2}{|c|}{ Fecha } & \multirow{2}{*}{ 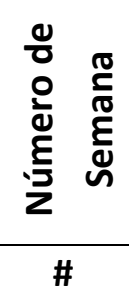 } & \multirow{2}{*}{ 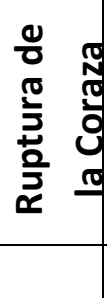 } & \multirow{2}{*}{ 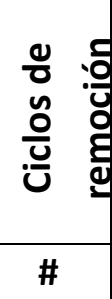 } & \multirow{2}{*}{ 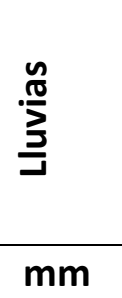 } & \multirow{2}{*}{ 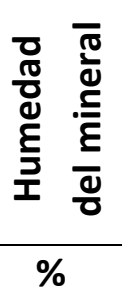 } & Proceso tecnológico \\
\hline Desde & Hasta & & & & & & Descripciones \\
\hline Abr-23 & Abr-28 & Inicio & No & 0 & 4,9 & 37,75 & Acarreo y Transporte \\
\hline $28 / 04 / 2014$ & $05 / 2014$ & 1ra & No & 10 & 17,2 & 34,77 & \multirow{7}{*}{$\begin{array}{l}\text { Remoción del mineral } \\
\text { según los requerimientos } \\
\text { de la tecnología y las } \\
\text { condiciones climáticas }\end{array}$} \\
\hline $05 / 05 / 2014$ & $12 / 05 / 2014$ & $2 d a$ & Si & 5 & 8,4 & 32,28 & \\
\hline $12 / 05 / 2014$ & $19 / 05 / 2014$ & $3 r a$ & $\mathrm{Si}$ & 10 & 6,1 & 30,12 & \\
\hline $19 / 05 / 2014$ & 26/05/2014 & 4 ta & $\mathrm{Si}$ & 8 & 7,9 & 28,19 & \\
\hline $26 / 05 / 2014$ & $02 / 06 / 2014$ & 5 ta & $\mathrm{Si}$ & 9 & 10 & 26,42 & \\
\hline $02 / 06 / 2014$ & $09 / 06 / 2014$ & 6 ta & $\mathrm{Si}$ & 4 & 91,9 & 24,93 & \\
\hline $09 / 06 / 2014$ & $16 / 06 / 2014$ & $7 \mathrm{ma}$ & $\mathrm{Si}$ & 10 & 42,1 & 23,57 & \\
\hline $16 / 06 / 2014$ & $23 / 06 / 2014$ & $8 v a$ & No & 0 & 0 & 23,51 & \multirow{5}{*}{$\begin{array}{l}\text { Determinación del } \\
\text { comportamiento del } \\
\text { mineral durante su } \\
\text { almacenaje o retención }\end{array}$} \\
\hline $23 / 06 / 2014$ & $30 / 06 / 2014$ & $9 n a$ & No & 0 & 0 & 23,41 & \\
\hline $30 / 06 / 2014$ & $07 / 07 / 2014$ & $10 \mathrm{ma}$ & No & 0 & 215,2 & 26,18 & \\
\hline 07/07/2014 & $14 / 07 / 2014$ & 11na & No & 0 & 169,3 & 27,02 & \\
\hline $14 / 07 / 2014$ & $21 / 07 / 2014$ & $12 \mathrm{ma}$ & $\mathrm{Si}$ & 5 & 0 & 26,60 & \\
\hline $21 / 07 / 2014$ & $23 / 07 / 2014$ & Final & Si & 5 & 0 & 26,81 & $\begin{array}{l}\text { Evacuación } \\
\text { transportación }\end{array}$ \\
\hline
\end{tabular}

\subsection{Formación de la coraza en el proceso de remoción}

Del 23 al 28 de abril se transportó el mineral desde los frentes mineros y se conformaron las pilas de mineral. Durante este periodo solo ocurrieron unos $5 \mathrm{~mm}$ de precipitaciones, el domingo 27 de abril, lo cual obligó a detener los trabajos por unos minutos por las dificultades para muestrear los camiones. En general hasta el 16 de junio solo se acumularon $188,5 \mathrm{~mm}$ de lluvias como se ilustra en la Figura 4, pero durante el proceso de almacenamiento entre el 17 de junio y el 17 de julio se produjeron $384 \mathrm{~mm}$ de lluvias. 


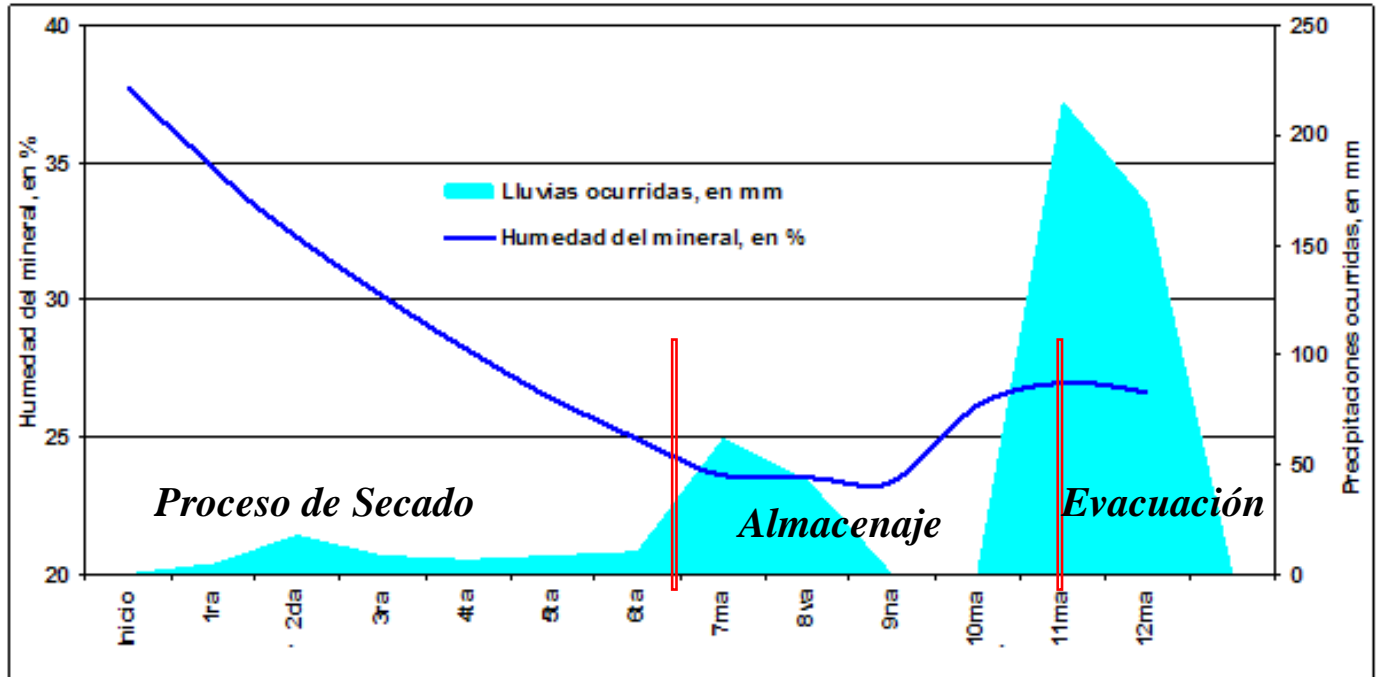

Figura 4: Reducción de la humedad del mineral durante las diferentes fases tecnológicas de la prueba de secado solar a la intemperie y su relación con las precipitaciones ocurridas durante cada periodo.

Como se puede apreciar, a pesar de las intensas lluvias ocurridas los días 3, 4, 7 y 8 de julio, la humedad del mineral se elevó ligeramente un $2.2 \%$ como resultado de la destrucción brusca de la coraza con el calentamiento diurno de los días $4,5,6$ y 7 de propio mes.

El espesor de la coraza depende de la humedad del mineral en el talud de la pila y de la intensidad de las lluvias.

Una vez que el mineral es secado prácticamente no absorbe agua nuevamente y las lluvias ocurridas se escurren en un elevado porcentaje a pesar del incremento de la superficie horizontal de la pila al disminuirse el ángulo de reposo del mineral con la reducción de la humedad en la pila, todo lo cual se debe a la formación de la coraza. Otro rasgo importante del proceso tecnológico se aprecia claramente, cuando el mineral se remueve, incrementa la absorción de energía y se produce la evaporación, pero cuando la pila de mineral deja de removerse, se reduce la evaporación significativamente, no disminuye la humedad, como ocurrió desde el 17 de junio hasta 17 de julio; mientras durante el proceso de evacuación, vuelve a producirse determinada reducción de humedad (Estenoz, 2011).

\subsection{Análisis de las condiciones climatológicas de la prueba}

En general las condiciones climáticas durante la prueba fueron típicas para esta época del año. Las intensas precipitaciones sucedidas durante el proceso de almacenamiento del mineral son típicas en términos más amplios para el micro-clima de la región de Moa, donde se han registrado picos de precipitaciones para 24 horas hasta de $1001 \mathrm{~mm}$.

Las precipitaciones sumaron durante el proceso de remoción del mineral los $188,5 \mathrm{~mm}$ de lluvias, lo cual significó que sobre la superficie de las pilas cayeron en ese periodo unas 157,6 
toneladas de agua. En la Figura 5 se puede observar el comportamiento de las precipitaciones y su influencia parcial sobre superficie de las pilas la humedad del mineral en las pilas.

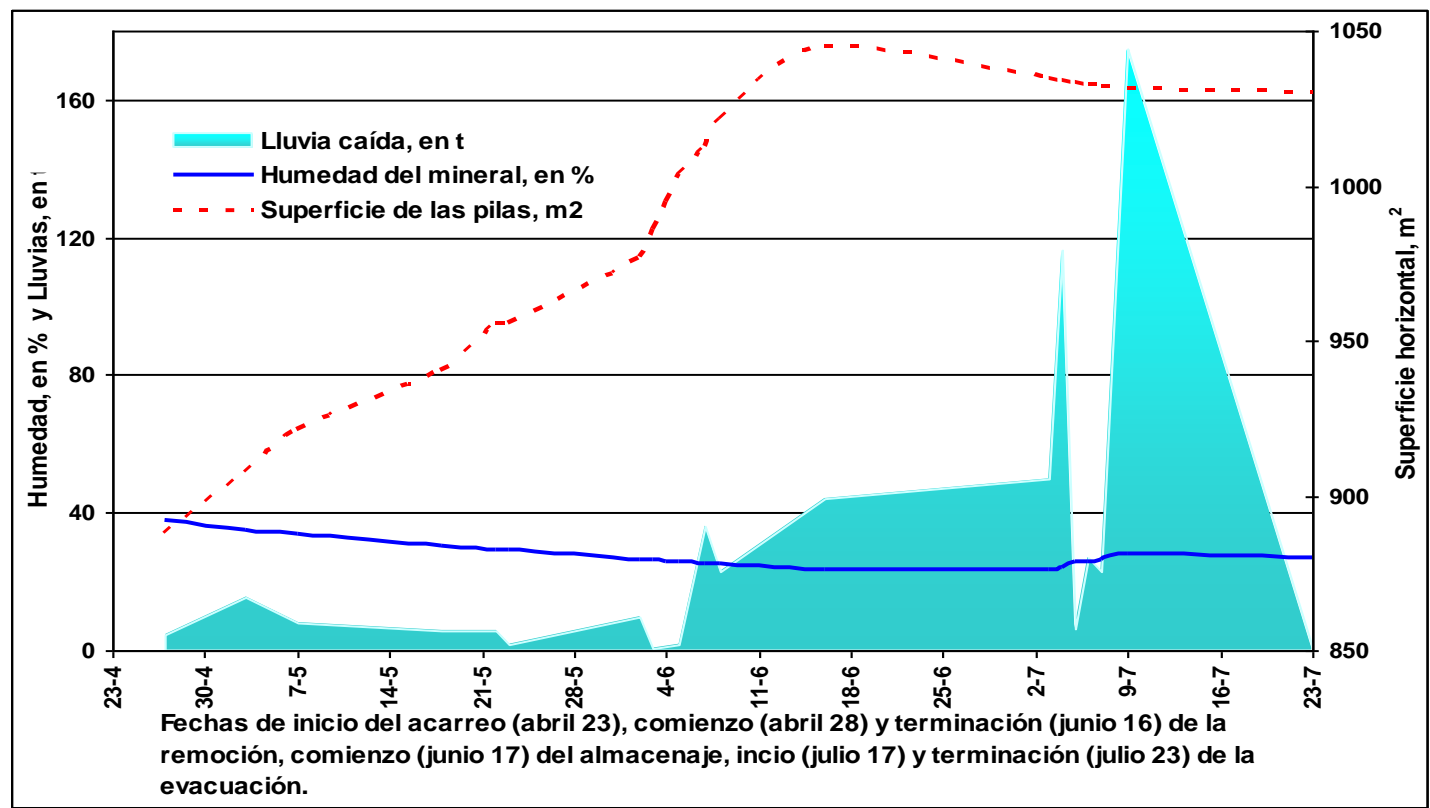

Figura 5: Comportamiento de la humedad del mineral y la influencia de las precipitaciones durante las diferentes etapas de la prueba de secado en dependencia de la superficie horizontal de las pilas.

Fue evaluado el potencial de secado del yacimiento considerando los resultados de las pruebas a escala semi-industrial en el Centro de Investigación del Níquel, entre los indicadores más significativos se consideraron los siguientes:

$\checkmark \quad$ Evaporación específica en los taludes según la orientación con respecto a la trayectoria del sol por el Zenit.

$\checkmark \quad$ Parámetros de las pilas formadas y removidas con cargador frontal.

$\checkmark \quad$ Utilización de retroexcavadoras, cargadores frontales o buldócer con rastrillos para variarles la altura desde 2,5 hasta $8,5 \mathrm{~m}$.

Tiempo de remoción considerando los regímenes medios de explotación del equipamiento minero y los cambios meteorológicos del microclima de Moa.

$\checkmark \quad$ Drenaje con calles perpendiculares y transversales entre las pilas de mineral.

$\checkmark \quad$ Traslado completo del borde inferior de un talud hasta el otro como culminación del proceso de remoción y secado del mineral.

$\checkmark \quad$ Remoción del mineral tres veces al día sobre los taludes por capas con espesor entre 15 y $30 \mathrm{~cm}$.

3.4 Modelación del proceso de secado en los depósitos 
Se programó en Micro Software Excel una hoja de cálculo enlazada a otras doce hojas donde se modelaron a partir de los datos obtenidos los fenómenos de remoción y secado de las pilas para cada una de las semanas de prueba. En la tabla 2 se muestra una parte de las modelos semanales.

Tabla 2. Procesos de remoción en el secado solar

\begin{tabular}{|c|c|c|c|c|c|c|c|}
\hline \multirow{2}{*}{ No } & \multirow[t]{2}{*}{ Parámetros } & \multirow[t]{2}{*}{ Índice } & \multirow[t]{2}{*}{ UM } & \multicolumn{4}{|c|}{ Pilas de mineral } \\
\hline & & & & $\mathbf{I}$ & II & III & IV \\
\hline 1 & Altura de la pila & $\mathrm{H}$ & $\mathrm{m}$ & 1.40 & 1.95 & 2.40 & 2.80 \\
\hline 2 & Humedad inicial del mineral en cada seccion & $\mathrm{Hi}$ & $\%$ & 37.62 & 37.76 & 37.78 & 37.83 \\
\hline 3 & Masa volumetrica del mineral en las pilas & Mv & $t / m^{3}$ & 1.4 & 1.4 & 1.4 & 1.4 \\
\hline 4 & Largo de las pilas & $\mathrm{L}$ & $\mathrm{m}$ & 140 & 140 & 140 & 140 \\
\hline 5 & Relacion talud de carga en remoción & Rto & $\mathrm{m}$ & 0.875 & 0.875 & 0.875 & 0.875 \\
\hline 6 & Relacion altura ancho de las pilas & Rhb & $\mathrm{m}$ & 0.875 & 0.875 & 0.875 & 0.875 \\
\hline 7 & Relacion talud de descarga en remocion & Rtd & $\mathrm{m}$ & 0.875 & 0.875 & 0.875 & 0.875 \\
\hline 8 & Ancho medio de la pila & $\mathrm{Bm}$ & $\mathrm{m}$ & 3.20 & 4.46 & 5.49 & 6.40 \\
\hline 9 & Semiancho por el talud de carga & $\mathrm{BC}$ & $\mathrm{m}$ & 1.60 & 2.23 & 2.74 & 3.20 \\
\hline 10 & Semiancho por el talud de descarga & $\mathrm{Bd}$ & $\mathrm{m}$ & 1.60 & 2.23 & 2.74 & 3.20 \\
\hline 11 & Ancho de las pilas en remocion & B & $\mathrm{m}$ & 3.20 & 4.46 & 5.49 & 6.40 \\
\hline 12 & Superficie horizontal total de las pilas & Shtp & $\mathrm{m}^{2}$ & & & & 887.59 \\
\hline 13 & $\begin{array}{l}\text { Lluvias caídas sobre las pilas sobre las pilas } \\
\text { de mineral }\end{array}$ & $\mathrm{LI}$ & $\mathrm{mm}$ & & & & 4.9 \\
\hline 14 & $\begin{array}{l}\text { Lluvias caídas sobre las pilas sobre las pilas } \\
\text { de mineral }\end{array}$ & $\mathrm{LI}$ & $\mathrm{t}$ & & & & 4.35 \\
\hline 15 & Aguas escurridas por la superficie de las pilas & Aet & $\mathrm{t}$ & & & & 1.22 \\
\hline 16 & Aguas escurridas por la superficie de las pilas & Aetp & $\%$ & & & & 28.14 \\
\hline 17 & Aguas retenidas en las pilas & Attp & $\mathrm{t}$ & & & & 3.13 \\
\hline 18 & Agua en el prisma I & $\mathrm{Ai}$ & $\mathrm{t}$ & 163.44 & & & \\
\hline 19 & Agua en el prisma II & Aii & $\mathrm{t}$ & & 152.82 & & \\
\hline 20 & Agua en el prisma III & Aiii & $\mathrm{t}$ & & & 161.50 & \\
\hline 21 & Agua en el prisma IV & Aiv & $\mathrm{t}$ & & & & 170.99 \\
\hline 22 & Relación agua vs \% de humedad & $\mathrm{RA} / \mathrm{H}$ & $t / \%$ & & & & 4.53 \\
\hline 23 & Incremento instantáneo de humedad & $\mathrm{IH}$ & $\%$ & & & & 0.69 \\
\hline 24 & Incrementos parciales de humedad & $\mathrm{IPH}$ & $\%$ & 0.00 & 0.01 & 0.03 & 0.08 \\
\hline 25 & Volumen total de las pilas en depositos & $\mathrm{Vt}$ & $\mathrm{m}^{3}$ & 310 & 599 & 905 & 1228 \\
\hline 26 & Masa minera humeda en la pila & $\mathrm{MH}$ & $\mathrm{t}$ & 434 & 839 & 1267 & 1719 \\
\hline 27 & Humedad inicial del mineral & $\mathrm{HI}$ & $\mathrm{t}$ & 163 & 317 & 479 & 650 \\
\hline 28 & Masa minera seca en la pila & MS & $\mathrm{t}$ & 271 & 522 & 788 & 1068 \\
\hline
\end{tabular}

La transformación de la pila durante el proceso de remoción se ilustra en la Figura 6 donde se simula aproximadamente el proceso de remoción del mineral con la pérdida de altura en los diferentes periodos de la prueba y la reducción del ángulo de los taludes. 


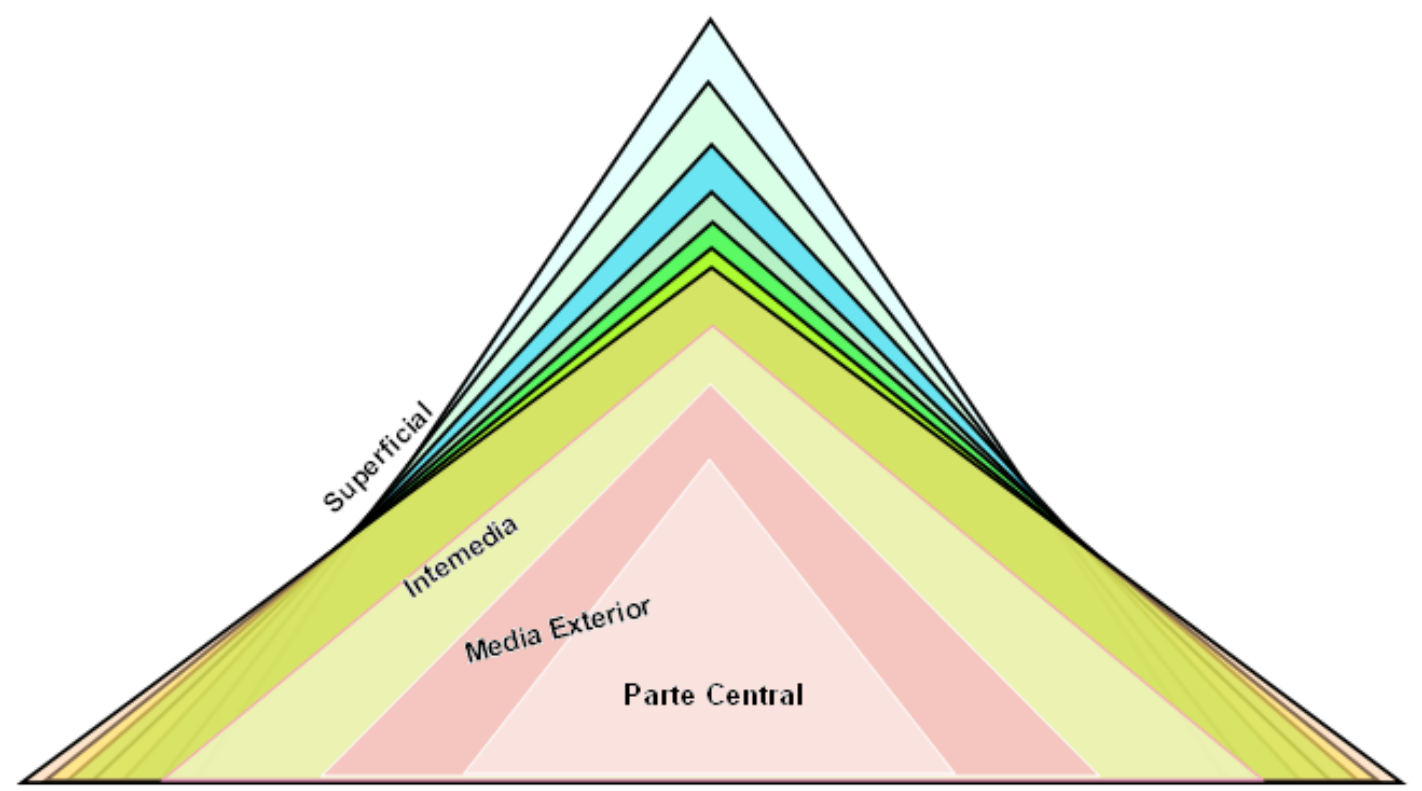

Figura 6: Proceso de descenso de la altura de las pilas durante la remoción del mineral y su influencia sobre el área horizontal de la pila, los ángulos de los taludes y el incremento de la capacidad de secado del mineral y la mejor formación de coraza para cobijar el mineral seco.

Se produce un aumento de la cantidad de mineral por superficie horizontal en los depósitos al disminuir el espacio entre pilas, dejando solo una pequeña zanja de unos $80-90 \mathrm{~cm}$ para la segregación, frenados y escurrimiento de las aguas y aumentar la cantidad de mineral protegido o recubierto por la coraza en las pilas, para la ocurrencia de intensas lluvias

\subsection{Estudio del Potencial de secado solar en la mina de la Empresa}

Fue realizado el estudio de la intensidad de la energía muestreada (Figura 7), en la prueba del Centro de Investigación del Níquel y la modelación de los datos meteorológicos medios anuales de la región de Moa.

Como se puede observar en la Figura 7 para secar anualmente 9 millones de toneladas de masa minera se requieren menos de 30 hectáreas de depósitos destinados al secado solar, lo cual es equivalente a un tercio de todas las áreas mineras disponibles en la mina (Miranda, 1996). Para reducir la humedad del mineral de $38 \%$ inicialmente hasta menos de $28,5 \%$ de salida de los depósitos, para lo cual solo se requerirían formar unas 60 pilas de mineral. 


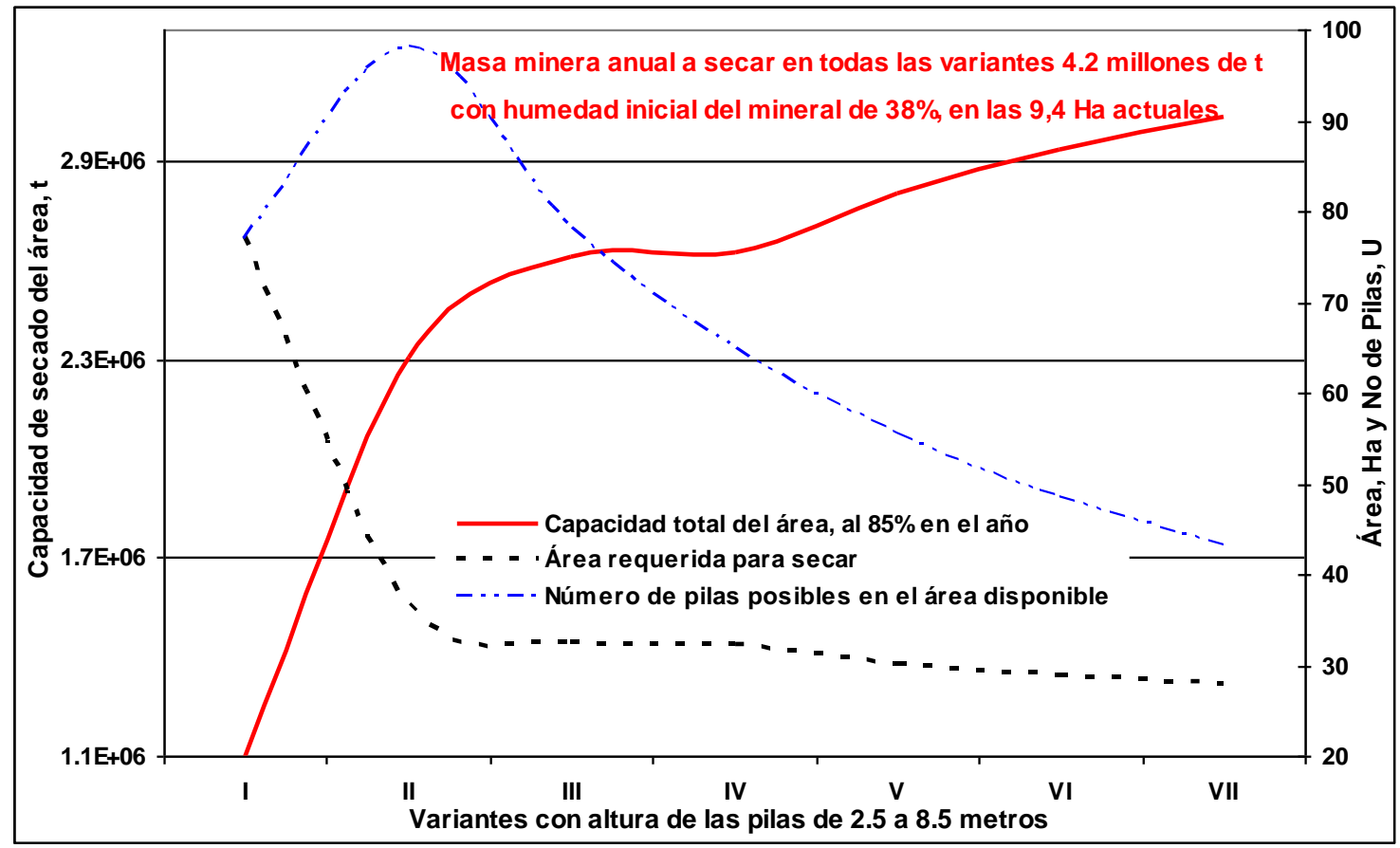

Figura 7: Potencial de secado solar en las áreas mineras actuales.

En la Figura 7 se puede observar la relación entre la altura de las pilas, la capacidad de secado del área actual y el área requerida para secar toda la masa minera que se alimente a la fábrica Empresa Comandante Ernesto Che Guevara, así como el número de pilas necesarias.

El estudio del potencial de secado solar en la mina de la Empresa, permitió obtener la relación de la variación de energía para el periodo estudiado en las condiciones de Moa y determinar los principales regímenes de secado en las plazoletas del Centro de Investigación del Níquel (Figura 8).

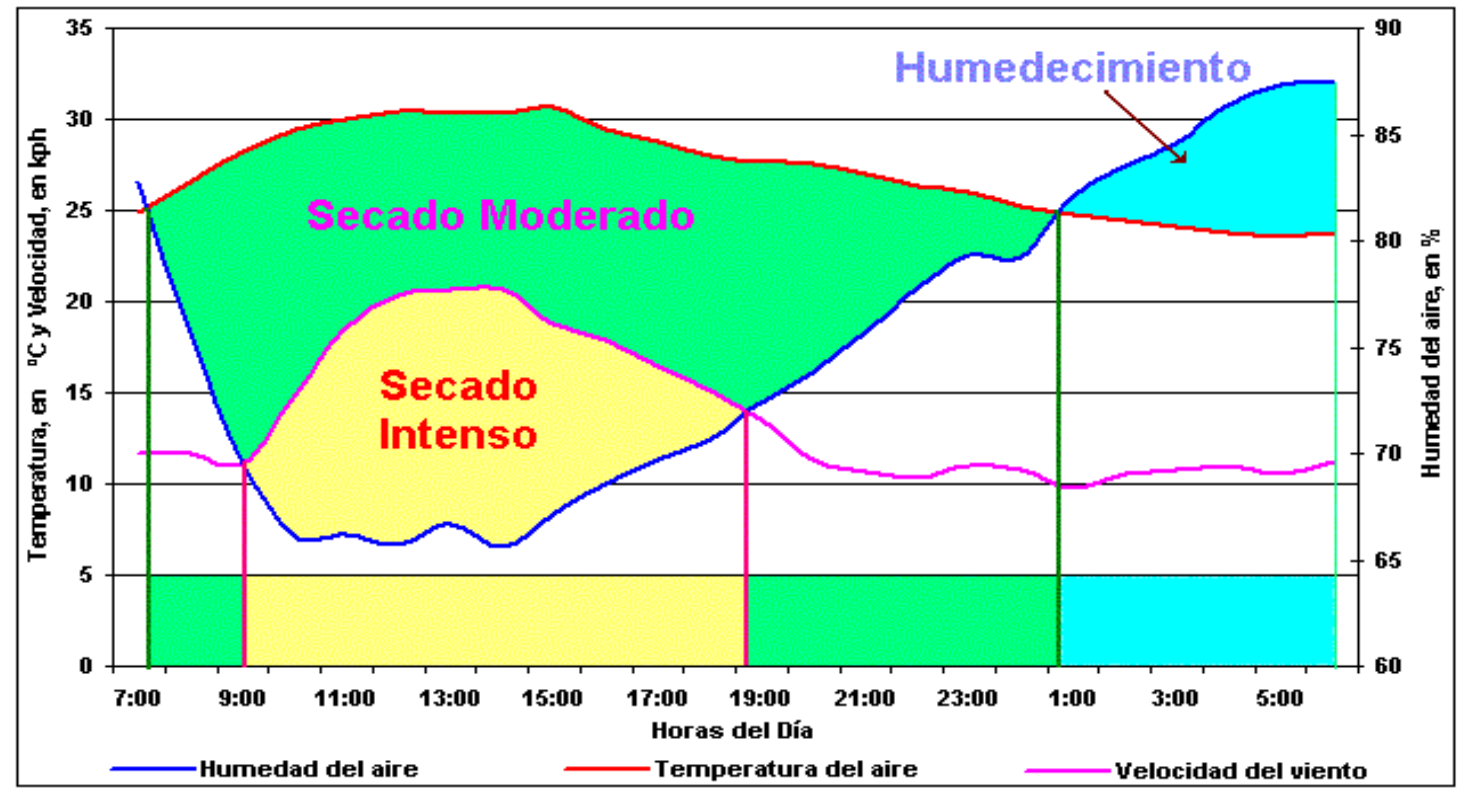

Figura 8: Relación de la variación de energía para el periodo estudiado en las condiciones de Moa y determinación de los principales regímenes de secado solar a la temperatura del aire. 
Con la ocurrencia de precipitaciones, se forma una coraza de 5 a $25 \mathrm{~mm}$ en la superficie del talud, el cual favorece el escurrimiento de las lluvias. El espesor de la coraza está relacionado con la humedad del mineral en el momento de ocurrencia de las precipitaciones. Mientras más seco se encuentra el mineral, mayor espesor y resistencia tiene la coraza que se forma con las precipitaciones y por ende, mayor cantidad de agua se escurre por los taludes de la pila.

Durante el periodo comprendido entre el 23 de abril y el 17 de julio, se sucedieron 543 milímetros de lluvia equivalentes a 539000 I de agua sobre la superficie horizontal de las pilas, de esa cantidad, por los taludes, se escurrieron unos 311000 I, equivalente al 57,7 \% de las lluvias ocurridas.

Con el transcurso de la prueba y el proceso de remoción del secado solar, tanto la altura como el ángulo de reposo, se fueron reduciendo hasta $1,9-2,1 \mathrm{~m}$ y $33-35^{0}$ respectivamente.

En la remoción de la capa seca de mineral, durante el proceso de secado, transcurría inicialmente entre 30-32 minutos y finalmente entre 26 y 28 minutos, lo cual está relacionado con la pérdida de altura y masa del mineral en las pilas durante el propio proceso de secado.

En el proceso de remoción del mineral en los taludes de las pilas, durante el secado solar, los terrones de mayor tamaño y aglomerados de minerales finos se desmenuzaron en pedazos más pequeños.

\section{CONCLUSIONES}

El desarrollo de esta investigación permitió realizar una evaluación del comportamiento del secado solar de lateritas a la intemperie, en condiciones de clima húmedo tropical, permitiendo reducir a valores inferiores de $\$ 0,09$, los costos por tonelada de mineral trasegado en los depósitos de la mina. Se incrementó la cantidad de mineral por superficie horizontal en los depósitos al disminuir el espacio entre pilas, dejando solo una pequeña zanja de unos $80-90 \mathrm{~cm}$ para la segregación, frenados y escurrimiento de las aguas y aumentar la cantidad de mineral protegido o recubierto por la coraza en las pilas, para la ocurrencia de intensas lluvias. Se obtuvo un valor promedio de humedad final equivalente a la evaporación del $40 \%$ del agua inicial. Se determinó que para alturas de pilas de 5,5 y 8,5 m se requieren 19 y 17 hectáreas respectivamente, para secar 4,2 millones de mineral en base húmeda, equivalente a la producción de $30000 \mathrm{t}$ anuales de níquel.

\section{REFERENCIAS BIBLIOGRÁFICAS}

Alepuz, H. (2001). Secado natural del mineral laterítico. X Simposio del CEINNIQ, Moa, Holguín, Cuba. (Memorias).

Estenoz, S., Espinosa, M. (2003). Procedimiento y equipo para secado solar de materiales a la intemperie, Solicitud de invención cubana No. 175, OCPI, OCPI, La Habana. 
Estenoz, S., Pérez, N. \& Rondón, E. (2004). Procedimiento de homogeneización y secado solar de materiales a granel e instalación requerida. Patente cubana No. 22883, OCPI, La Habana, Cuba.

Estenoz, S. (2007). Evaluación de las tecnologías de explotación de depósitos mineros para mezclas, beneficio, homogeneización y secado solar en la mina de la ECECG, ECG, UBMina, Informe Técnico, pp. 46.

Estenoz, S. (2011). Procedimiento tecnológico para beneficio, secado solar y homogenización del mineral laterítico. Disponible en: www.redciencia.cu/geobiblio/paper/2011 Estenoz MIN1011.pdf

García, F. (2014). Evaluación de los efectos del proceso de secado sobre la calidad de la mena lateritica. Disponible en: www.bdigital.unal.edu.co/42012/1/822137.2014.pdf

Marcilli, Y. (2013). Perfeccionamiento del diseño de los depósitos mineros de la empresa Ernesto Che Guevara. Disponible en: www.redciencia.cu/geobiblio/paper/2013 Mustelier MIN1017.pdf

Miranda, J. (1996). Composición y conjugación de métodos de determinación de humedad de la industria del Níquel. Minería y Geología. 13(2), pp. 39-45

Retirado, Y. (2007). Comportamiento de la humedad durante el secado solar del mineral laterítico. Disponible en: https://revista.ismm.edu.cu/index.php/revistamg/article/download/83/88

Retirado, Y. (2012). Optimización del secado solar del mineral laterítico en la industria cubana del níquel. 28(2), $\quad$ pp. 30-46. Disponible https://revista.ismm.edu.cu/index.php/revistamg/search/authors/view?firstName=Yoalbys\& middleName $=$ \&lastName $=$ RetiradoMediaceja\&affiliation=Instituto\%20Superior\%20Minero\%20Metal\%C3\%BArgico\&country=CU

Retirado, Y. (2015). Velocidad de secado en pilas del mineral laterítico almacenadas a la intemperie. 31(3), pp.

45-61 Ricardo, R., Vargas, A. (2014). Velocidad de secado del carbonato de níquel. Revista Tecnología Química. 34(2), pp. 34-45. Disponible en:

http//www. scielo.sld.cu/scielo.php?script=sci_arttext\&pid=\$2224-61852014000200003

Romero, Y. (2006). Estudio experimental a escala piloto del secado solar del mineral laterítico. Trabajo de Diploma. ISMM, pp. 55. 